\title{
DISCLAIMER
}

This report was prepared as an account of work sponsored by an agency of the United States Government. Neither the United States Government nor any agency thereof, nor any of their employees, makes any warranty, express or implied, or assumes any legal liability or responsibility for the accuracy, completeness, or usefulness of any information, apparatus, product, or process disclosed, or represents that its use would not infringe privately owned rights. Reference herein to any specific commercial product, process, or service by trade name, trademark, manufacturer, or otherwise does not necessarily constitute or imply its endorsement, recommendation, or favoring by the United States Government or any agency thereof. The views and opinions of authors expressed herein do not necessarily state or reflect those of the United States Government or any agency thereof.

\section{INTERACTING BOSON MODEL: SELECTED RECENT DEVELOPMENTS*}

\author{
A. B. Balantekin ${ }^{\dagger}$ \\ Physics Division, Oak Ridge National Laboratory \\ Oak Ridge, TN 37831 U.S.A.
}

to be presented at

\section{Second Conference on the Intersections Between}

Particle and Nuclear Physics

Lake Louise, Canada

May $26-30,1986$

\footnotetext{
* Research sponsored by the Division of Nuclear Physics, U.S. Department of Energy under contract DE-AC05-840R21400 with Martin Marietta Energy Systems, Inc.

†ugene P. Wigner Fellow. 


\title{
INTERACTING BOSON MODEL: SELECTED RECENT DEVELOPMENTS ${ }^{*}$
}

\author{
A. B. Balantekin ${ }^{\dagger}$ \\ Physics Division, Oak Rtdge National Laboratory \\ Oak RIdge, TN 37831 U.S.A.
}

\begin{abstract}
The Interacting Boson Model is briefly reviewed. Recent app11cations of this model to the low-lying collective magnetic-dipole excitations and to the spectra of $195 \mathrm{Ir}$ are described.

\section{INTRODUCTION}

Irteractinig Boson Model is an algebralc approach to even-even nuclel formulated in terms of bosonic variables with emphasis being placed on the symetries assoctated with the quadrupole collectiv1ty. I In this model, low-lying energy levels are generated as states of a system of $N$ bosons carrying angular momentum $L=0$ or $L=2$. $N$ Is taken to be the number of correlated pairs of valence neutrons or pairs of valence protons. The palrs with $L=0$ are similar to Cooper pairs in a gas of electrons. Introducing creation and annihilation operators for six types of bosons, $b_{i}, b_{f}^{\dagger}$, one obtains a bosonic realization of the $\mathrm{U}(6)$ algebra, $G_{1 j}=b_{1} b_{j}$. The one-boson terms of the Hamiltonian are written as a linear combination of the $U(6)$ generators and the boson-boson interaction terms as a combination of quadratic products of these generators:

$$
H=\sum \varepsilon_{1 j} G_{1 j}+\sum u_{1 j k \ell} G_{1 j} G_{k \ell}
$$

The coefficlents in the above equation are free parameters to be fixed separately for each nucleus, subject to the restriction that the total angular momenta of the bosons is conserved. Eigenstates of the Hamiltonian are constructed by the application of boson creation operators on a vacuum $\mathrm{N}-\mathrm{times}$

$$
b_{1}^{\dagger} \ldots \ldots b_{1_{N}}^{\dagger} \mid 0>
$$

1.e., one forms the completely symmetric representations of $U(6)$. $\mathbf{I n}_{\mathbf{1 1}}$ general, such states are labeled by the boson number $N$ and by the labels of all the representations of all possible subalgebras, included in the completely symmetric representation of $U(6)$. Hence,

\footnotetext{
*Research sponsored by the Division of Nuclear Physics, U.S. Department of Energy under contract DE-AC05-840R21400 with Martin Marietta Energy Systems, Inc.

†Eugene P. Wigner Fellow.
} 
for most nuclel, the diagonalization of the Hamlitonian must be performed numerically. However, the popularity that the Interacting Boson Model has enjoyed in recent years is mostly facilitated by the existence of analytically solvable limits.

Analytical solutions are possible when the free parameters take special values such that the first term In Eq. (1) can be written as a combination of IInear Casimir uperators and the second term as a combination of quadratic Casimir operators of the algebra $U(6)$ and Its subalgebras. In such cases the quantum numbers are given by the labels associated with the algebras in a particular chain starting with $U(6)$ and ending with $S O(3)$, and the Hamiltonian is sald to possess a dynamical symmetry. This situation is reminiscent of the Gell-Mann-Okubo SU(3) mass formula

$$
E\left(I, I_{3}, Y\right)=E+a Y+b\left[\left(Y^{2} / 4\right)-I(I+1)\right]
$$

which 18 associated with the group chain

$$
\operatorname{SU}(3) \supset \operatorname{SU}(2) \times U(1) \supset \operatorname{SO}(2) \times U(1) .
$$

There are three possible dynamical symmetries for the Interacting Boson Model Hamiltonian with the second group in the chain being $\mathrm{SU}(5), \mathrm{SU}(3)$, or $\mathrm{SO}(6)=$

The existence of dynamical symmetries of the Interacting Boson Model Hamiltonian Implies the existence of analytical expressions not only for energy $\in$ igenvalues, but also for the eigenstates. Consequently, to establish whether or not a particular dynamical symmetry is physically realized, it is not sufficlent to compare energy spectra. It is essential to test predictions for the electromagnetic transition rates, which are calculated using the wavefunctions. The dynamical symmetries of the Interacting Boson Model provide a simple framework to analyze and classify the experimental data and have been successfully used in this context during the last ten years. 1 In this talk, I highlight two of the recent developments in the applicatIons of the Interacting Boson Model.

\section{LOW-LYING COLLECTIVE MAGNETIC-DIPOLE EXCITATION MODE}

There 1s a second version of the Interacting Boson Model, which is much richer in structure than the version discussed in the Introduction. If no distinction is made between bosons which are correlated pairs of neutrons and bosons which are correlated pairs of protons, then the wavefunction given in $\mathrm{Eq}$. (2) contains only the completely symmetric representations of $U(6)$. However, if such a distinction is made, the states which are partially antisymnetrized between neutron and proton bosons should also be experimentally observable. This new version of the model is called the neutron-proton Interacting Boson Model.

Semiclassically, a deformed nucleus can be visualized as an ellipsoid Inside of which protons and neutrons are uniformly distributed. When such a nucleus is placed in an external field, the proton ellipsold translationally osclllates around the neutron 
ell1psold. The direction of the vibration can be efther parallel or perpendtcular to the major axis of the deformed nucleus. Consequently, a double-humped strength distribution can be experimentally observed. This is the well-known soldhaber-Teller mode. For two separate ellipsoidal rigid bodies, which are slightly tilted away from each other, a different kind of oscillation is also possible where the two shapes wobble around the same $\mathrm{axis}^{2}$ as depicted In Fig. 1. Such a motion would excite a $\mathrm{J}^{\mathrm{m}}=\mathrm{1}^{+}$state through the orbital current part of the Ml operator. One would expect that the energy of such a state 1 s comparable to the energy of the El giant resonance (the Goldhaber-Teller mode).

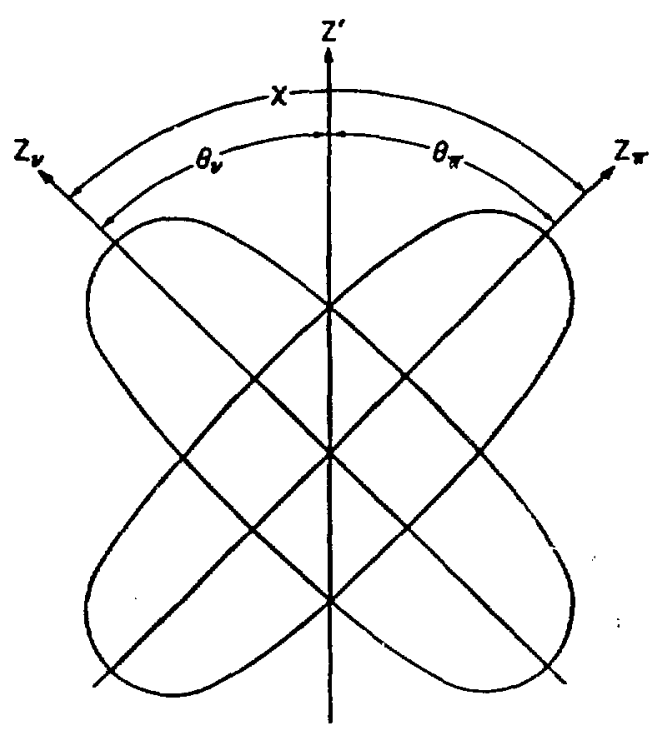

F1g. 1. Classical picture of the collective $1^{+}$configuration
A collective magneticdipole excited $1^{+}$state has been discovered ${ }^{3}$ in electron scattering first over ${ }^{156} \mathrm{Gd}$ targets and later other nuclei in this region. The excitation energy in this mass region was found to be approxImately $3 \mathrm{MeV}$. Recently $\left(Y, \gamma^{\prime}\right)$ experiments on a series of $\mathrm{Gd}$ isotopes has confirmed ${ }^{4}$ the energies and more importantly the spin of these states. This excitation energy is much lower than one would expect for the wobble mode discussed in the previous paragraph, where the entire nuclear system participates. However, the mixed symmetry states of the neutron-proton Interacting Boson Model in the SU(3) limit give rise $^{5}$ to a low-lying $\mathrm{K}^{\pi}=1^{+}$band, where the $\mathrm{Ml}$ matrix element connecting the

ground state and the bandhead is very large. Since in this model only the valence nucleons contribute to the wobble motion, as opposed to the entire nuclear system, the excitation energy would be relatively low. Furthermore, one can show ${ }^{6}$ that in the classical limit of the neutron-proton Interacting Boson Model Hamiltonian these $1^{+}$ states can be regarded as small amplitude oscillations of the angle between the two symmetry axes of the deformed valence neutrons and protons. Consequently, one can interpret the experimentally observed $1^{+}$state in ${ }^{156} \mathrm{Gd}$ as a neutron-proton antisymmetric state in the Interacting Boson Model framework.

After the inftal discovery in ${ }^{156} \mathrm{Gd}$, the new collective 1sovector magnetic dipole mode has been observed in a number of nucle1.7 One should stress that this new mode is not an isolated example, but the prototype of an entire new class of states which occur at that 
energy and have collectlve properties.8 Since these states are neutron-proton antisymmetric, thel $r$ experimental study should yleld a better understanding of the neutron proton forces in the collective models.

\section{DESCRIPTION OF THE NUCLEUS 195 Ir IN THE FRAMEWORK OF U(6/4) SUPERSYMMETRY}

In order to study odd-even nuclel, where one of the nucleons is unpalred, In a framework similar to the Interacting Boson Model, fermionlc degrees of freedom should be explicltly introduced. Such an extended model is the Interacting Boson-Fermion Mode1.9 If the angular momentum of the fermion has some speciflc value, and furthernore If the boson-boson and boson-fermion interactions have particular forms, one can also obtain analytical expressions for the energles and transition rates. An experimentally relevant example is provided when the bosonic core is described by the $O(6)$ dynamical symmetry of the Interacting Boson Model and when $f=3 / 2$ for the unpalred fermion, since $f=3 / 2$ is the angular momentum in the $S U(2)$ decomposition of the lowest-dimensional spinor representation of $n(6)$. In this scheme, bosonic states ( $1 . e$. those in the even-even nucleus) are transformed by the tensor representations of $O(6)$ and fermionic atates ( $1 . e$. those in the nelghboring odd-even nucleus) are transformed by the spinor representations, which are associated with the universal covering group Spin(6). Such symmetries were termed as Bose-Fermi symmetries. 10

In principle, the spectra of the even-even and the odd-even nuclel can be fitted separately. However, in certain cases the parameters appearing in the Hamiltonian and in the electromagnetic transition operators take the same value for both kinds of nuclei. In such cases, a supersymmetry is realized. Using the 1somorphism Spin(6) SU(4), the scheme described in the previous paragraph can be embedded in the supergroup SU(6/4). Th1s supersymmetry has been observed $^{11}$ in the Os-Ir regton where a number of nuclel are placed in the same supermultiplet (FIg. 2). There are other examples of approximate supersymetries in nature, one of them being the equivalence of the slopes of the Regge trajectorles for mesons and baryons. Indeed, the $U(N / M)$ type supergroups were first introduced in an effort 12 to explain this equivalence. The prominence of the nuclear supersymmetrles, however, is that thelr wel1-defined predictions can be thoroughly tested in the laboratory. In the rest of this talk, I describe such a recent test.

There is a simpler description of the odd-even nuclei, called the weak coupling model. In this model the angular momentum of the fermion is directly coupled to the states $L=0,2, \ldots$ in the eveneven core and the resulting states are split by a $\mathrm{J}(\mathrm{J}+1)$ interaction. In the Spin(6) scheme the $3 / 2$ member of the $3 / 2 \times 2$ multiplet is at an energy higher than that is expected from the simple-minded weak coupling picture. Since this state is generated by the quadrupole Interaction between the bosons and the fermion, it does not belong to the same SpIn(6) representation as the ground state. Consequently, a precise measurement of the position of this sitate is requisite to 


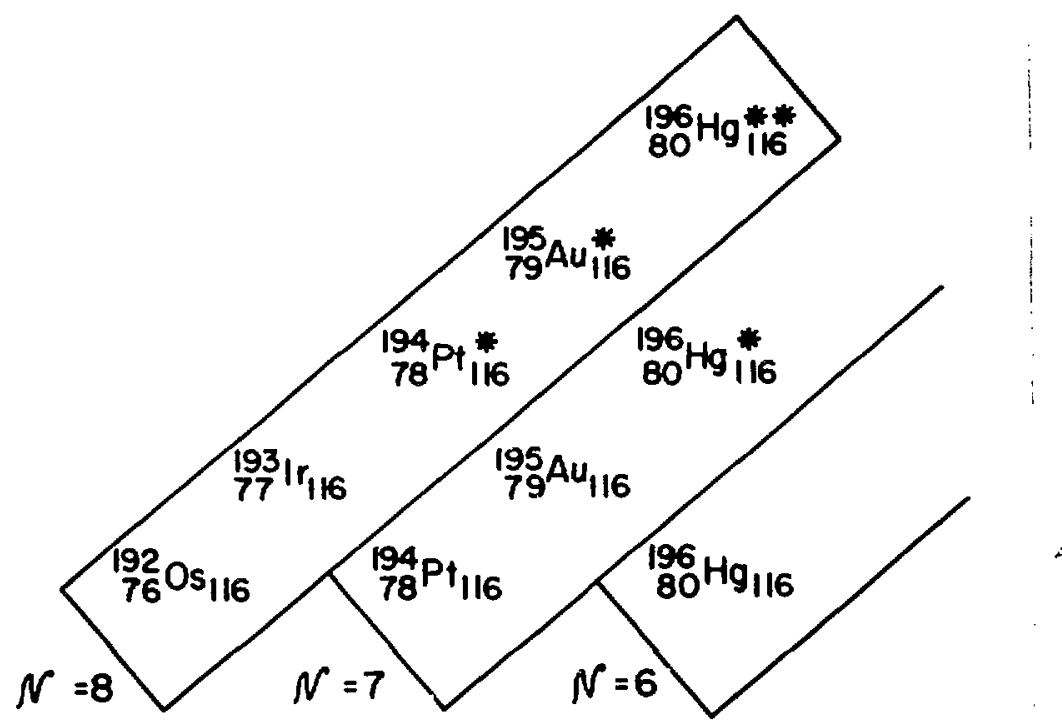

F1g. 2. Supersymmetric multiplets in the Os,Pt region.

establish the applicability of the Spln(6) Bose-Fermi symmetry.

Based on previous work in this region, ${ }^{195} \operatorname{Ir}$ is expected to be a good example of Spin(6) symmetry. The low-lying positive parity states and their electromagnetic de-excitations have recently been studied ${ }^{13}$ in the ${ }^{193} \operatorname{Ir}(2 n, Y)^{195} \operatorname{Ir}$ reaction. Using the observed $E 2$ transition, the $3 / 2$ state mentioned in the preceding paragraph has been locater at $286 \mathrm{keV}$. In ${ }^{195} \mathrm{Ir}$, the ground state is located in the $\sigma=13 / 2$ representation of $\operatorname{Spln}(6)$, and the $286-\mathrm{keV} 3 / 2$ state is the head of the $\sigma=11 / 2$ representation. Furthermore, using the parameters obtalned for the nucleus 1940 , one can predict the spectra of ${ }^{195} \mathrm{Ir}$ by invoking the $U(6 / 4)$ Spin(6) supersymmetry. The resulting predictions are compared with the experimental level scheme In Fig. 3, taken from Cizewsk1, et al.13 The sallent feature of this figure is the remarkable regularity in the repetition of the $\sigma$ sequences, which is a unique signature of the supersymmetry scheme.

I would like to thank J. Clzewskl for communicating her recent results prior to publication.

\section{REFERENCES}

1. For recent reviews see A. Arima and F. Iache11o, Adv. Nucl. Phys. 13, 139 (1984); A.E.L. Dieperink and G. Wenes, Ann. Rev. Nucl. Part. Sci. 35, (1985).

2. N. Lo Iudice and F. Palumbo, Phys. Rev. Lett. 41, 1532 (1978).

3. D. Bohle et al., Phys. Lett. 137B, 27 (1984).

4. U.E.P Berg et a1., Phys. Lett. 149B 59 (1984).

5. F. Iachello, Nucl. Phys. A358, $\overline{89 \mathrm{c}}$ (1981); A.E.L. Dleperink, Prog. In Part. and Nucl. Phys. 9, 121 (1983).

6. A. B. Balantekin and B. R. Barrett, Phys. Rev. C32, 288 (1985); S. PIttel and J. Dukelsky, 1bid. 335 (1985); R. $\overline{B 1 j k e r, ~ 1 b i d . ~}$ 
1442 (1985); N. R. Walet, P. J. Brussaard, and A.E.L. Dieperink, Phys. Lett. 163B, 1 (1985).

7. For a recent review, see A. Richter, in Nuclear Structure 1985, ed. by R. Brogl1a, G. B. Hagemann, and B. Hersk1nd (ElsevierNorth-Holland, Amsterdam, 1985).

8. F. Iache11o, Phys. Rev. Lett. 53, 1427 (1984).

9. For a recent review, see 0. Scholten, Prog. In Part. and Nucl. Phys. 14, 189 (1985).

10. F. Iachello, Phys. Rev. Lett. 44, 772 (1980).

11. A. B. Balantek1n, I. Bars, and $\bar{F}$. Iachel1o, Phys. Rev . Lett. 47, $19(1981)$.

12. H. Myazawa, Phys. Rev. 170, 1586 (1968).

13. J. A. Cizewski, et al., Yale preprint YALE-3074-854a (1986).

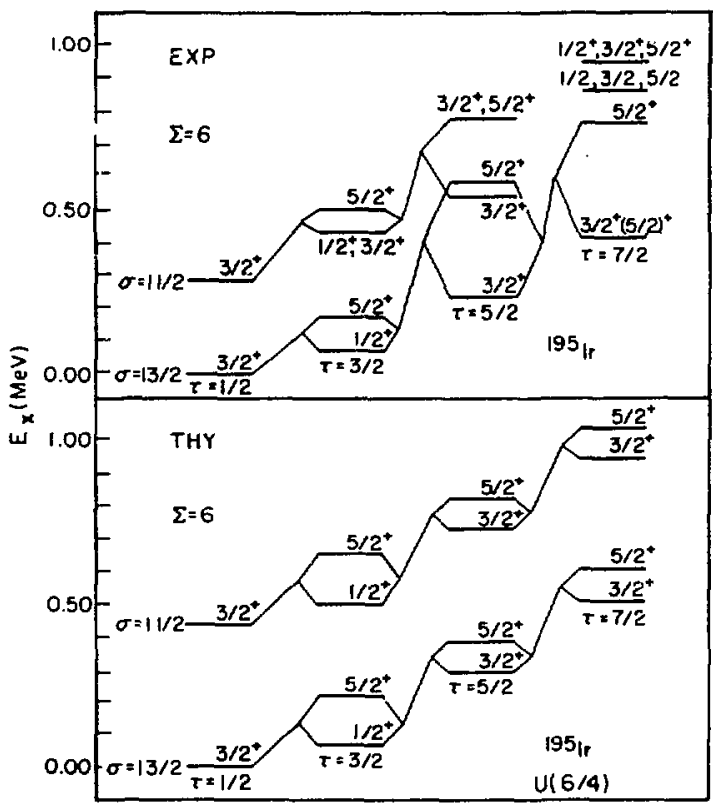

Fig. 3. Comparison between supersymmetry predictions and positive-partity states in ${ }^{195}$ Ir (from Ref. 13). 